\title{
The nature and oxidative reactivity of urban magnetic nanoparticle dust provide new insights into potential neurotoxicity
}

Xiangxing Long, ${ }^{1,2}$ Yi-hao Luo, ${ }^{2}$ Zhaobo Zhang, ${ }^{3}$ Chenwei Zheng, ${ }^{2}$ Chao Zeng, ${ }^{1}$ Yuqiang Bi, ${ }^{1}$ Chen Zhou, ${ }^{2}$ Bruce E. Rittmann, ${ }^{2}$ T. David Waite, ${ }^{4}$ Pierre Herckes ${ }^{3}$ and Paul Westerhoff ${ }^{\star 1}$

${ }^{1}$ Nanosystems Engineering Research Center for Nanotechnology-Enabled Water Treatment, School of Sustainable Engineering and the Built Environment, Arizona State University, Tempe, AZ 85287-3005, USA

${ }^{2}$ Biodesign Swette Center for Environmental Biotechnology, Arizona State University, Tempe, AZ 85287-5701, USA

${ }^{3}$ School of Molecular Sciences, Arizona State University, Tempe, AZ, 85287-1604, USA

${ }^{4}$ Water Research Center, School of Civil and Environmental Engineering, The University of New South Wales, Sydney, NSW 2052, Australia

* Corresponding Author: Arizona State University, Box 3005, Tempe, AZ 85287-3005; phone: 480-965-2885; email: p.westerhoff@asu.edu

The Supplemental information includes:

Number of pages: 7

Supplemental Texts: S1

Supplemental Tables: S2-S3

Supplemental Figures: S3-S6

Supplemental References: S7 
Supplementary Information

Table S1: an extended list of components detected in ICP-MS of magnetite nanoparticles and magnetic dust.

\begin{tabular}{|c|c|c|c|c|}
\hline Element & unit & $\begin{array}{l}\text { Urban magnetic } \\
\text { dust }\end{array}$ & $\begin{array}{l}\text { Commercial } \\
\text { magnetite } \\
\text { nanoparticles }\end{array}$ & Neurotoxicological effects \\
\hline $\mathrm{Fe}$ & $\mathrm{mg} / \mathrm{g}$ & $636 \pm 2$ & $540 \pm 0.8$ & Cognitive decline. ${ }^{1}$ \\
\hline $\mathrm{Mn}$ & $\mathrm{mg} / \mathrm{g}$ & $4.086 \pm 0.005$ & $2.78 \pm 0.02$ & $\begin{array}{l}\text { Potential neuronal cell death and inflammation } \\
\text { due to manganism. }{ }^{2}\end{array}$ \\
\hline $\mathrm{Zn}$ & $\mathrm{mg} / \mathrm{g}$ & $1.5 \pm 0.8$ & $0.2 \pm 0.4$ & $\begin{array}{l}\text { Excess } Z n \text { disrupts zinc homeostasis in brain can } \\
\text { causes ferroptosis. }\end{array}$ \\
\hline $\mathrm{Cu}$ & $\mathrm{mg} / \mathrm{g}$ & $1.180 \pm 0.006$ & $0.148 \pm 0.004$ & $\begin{array}{l}\text { Cu causes aggregation of neurodegenerative } \\
\text { proteins and brain oxidative stress. }{ }^{4}\end{array}$ \\
\hline $\mathrm{Cd}$ & $\mu \mathrm{g} / \mathrm{g}$ & $1.8 \pm 0.7$ & $1.2 \pm 0.3$ & $\begin{array}{l}\text { Cd disrupts the permeability of blood brain } \\
\text { barrier. }^{5}\end{array}$ \\
\hline $\mathrm{Pb}$ & $\mu \mathrm{g} / \mathrm{g}$ & $12 \pm 1$ & $32.4 \pm 0.5$ & $\begin{array}{l}\mathrm{Pb} \text { accumulation in hippocapus causes memory } \\
\text { loss and intelligence deficits. }{ }^{6}\end{array}$ \\
\hline $\mathrm{TI}$ & $\mu g / g$ & $2 \pm 1$ & $1.0 \pm 0.9$ & $\mathrm{TI}$ causes disorders in central nervous system. ${ }^{7,8}$ \\
\hline $\mathrm{Mg}$ & $\mathrm{mg} / \mathrm{g}$ & $1.49 \pm 0.01$ & $1.82 \pm 0.01$ & Not reported yet \\
\hline $\mathrm{Cr}$ & $\mathrm{mg} / \mathrm{g}$ & $1.30 \pm 0.01$ & $1.06 \pm 0.03$ & Not reported yet \\
\hline B & $\mathrm{mg} / \mathrm{g}$ & $1.1 \pm 0.3$ & $0.4 \pm 0.2$ & Not reported yet \\
\hline Mo & $\mathrm{mg} / \mathrm{g}$ & $0.7 \pm 0.3$ & $0.13 \pm 0.06$ & Not reported yet \\
\hline v & $\mathrm{mg} / \mathrm{g}$ & $0.586 \pm 0.007$ & $0.03 \pm 0.02$ & Not reported yet \\
\hline $\mathrm{Ni}$ & $\mathrm{mg} / \mathrm{g}$ & $0.42 \pm 0.01$ & $0.350 \pm 0.005$ & Not reported yet \\
\hline $\mathrm{Ca}$ & $\mathrm{mg} / \mathrm{g}$ & $0.33 \pm 0.09$ & $0.004 \pm 0.006$ & Not reported yet \\
\hline$P$ & $\mathrm{mg} / \mathrm{g}$ & $0.3 \pm 0.1$ & $0.4 \pm 0.3$ & Not reported yet \\
\hline $\mathrm{Sb}$ & $\mathrm{mg} / \mathrm{g}$ & $0.20 \pm 0.09$ & $0.04 \pm 0.02$ & Not reported yet \\
\hline $\mathrm{Ba}$ & $\mathrm{mg} / \mathrm{g}$ & $0.1860 \pm 0.0005$ & $0.0004 \pm 0.0004$ & Not reported yet \\
\hline $\mathrm{Sr}$ & $\mathrm{mg} / \mathrm{g}$ & $0.1630 \pm 0.0007$ & $0.0059 \pm 0.0009$ & Not reported yet \\
\hline Sn & $\mathrm{mg} / \mathrm{g}$ & $0.12 \pm 0.01$ & $0.010 \pm 0.004$ & Not reported yet \\
\hline Co & $\mathrm{mg} / \mathrm{g}$ & $0.12 \pm 0.02$ & $0.07 \pm 0.01$ & Not reported yet \\
\hline $\mathrm{Li}$ & $\mathrm{mg} / \mathrm{g}$ & $0.10 \pm 0.04$ & $0.025 \pm 0.015$ & Not reported yet \\
\hline Se & $\mu \mathrm{g} / \mathrm{g}$ & $73 \pm 65$ & $22 \pm 26$ & Not reported yet \\
\hline $\mathrm{Na}$ & $\mu \mathrm{g} / \mathrm{g}$ & $40 \pm 68$ & $396 \pm 78$ & Not reported yet \\
\hline $\mathrm{Ag}$ & $\mu g / g$ & $34 \pm 17$ & $8 \pm 5$ & Not reported yet \\
\hline $\mathrm{Be}$ & $\mu \mathrm{g} / \mathrm{g}$ & $28 \pm 10$ & $16 \pm 8$ & Not reported yet \\
\hline $\mathrm{Au}$ & $\mu g / g$ & $24 \pm 14$ & $9 \pm 3$ & Not reported yet \\
\hline $\mathrm{Pd}$ & $\mu \mathrm{g} / \mathrm{g}$ & $3 \pm 1$ & $0.8 \pm 0.3$ & Not reported yet \\
\hline
\end{tabular}




\section{Supplementary Information}

Table S2: Major potentially neurotoxic metals detected in AA using ICP-MS. The samples were taken from the supernatant of vials running AA assay. BDL indicates below detection limit.

\begin{tabular}{ccc}
\hline \multicolumn{2}{c}{ Potentially neurotoxic metals detected in AA assay } \\
(ppb)
\end{tabular}

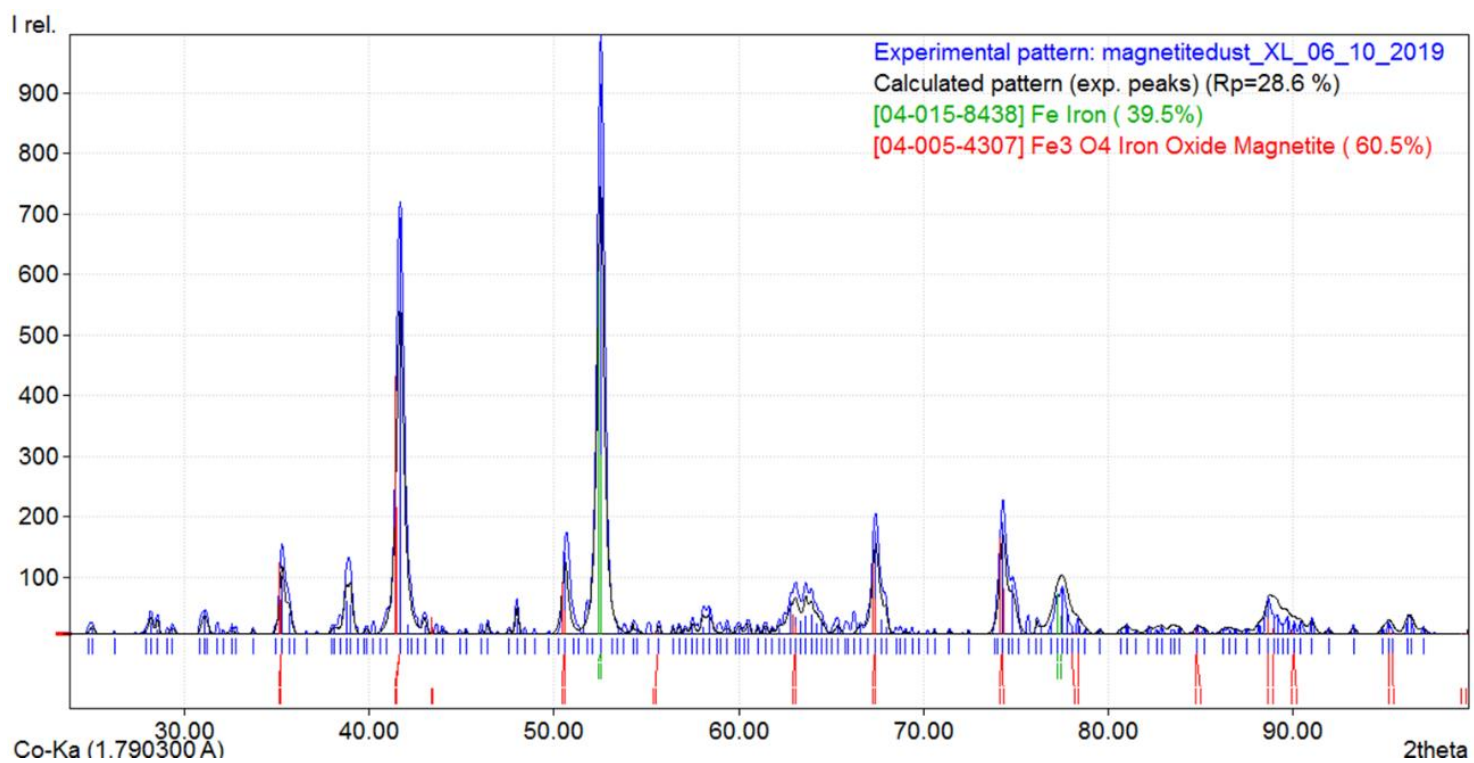

Figure S1: XRD elemental estimation of magnetic dust using Match! 2 software. The magnetic dust particles contain approximately $40 \%$ elemental iron and $60 \%$ magnetite. 


\section{Supplementary Information}

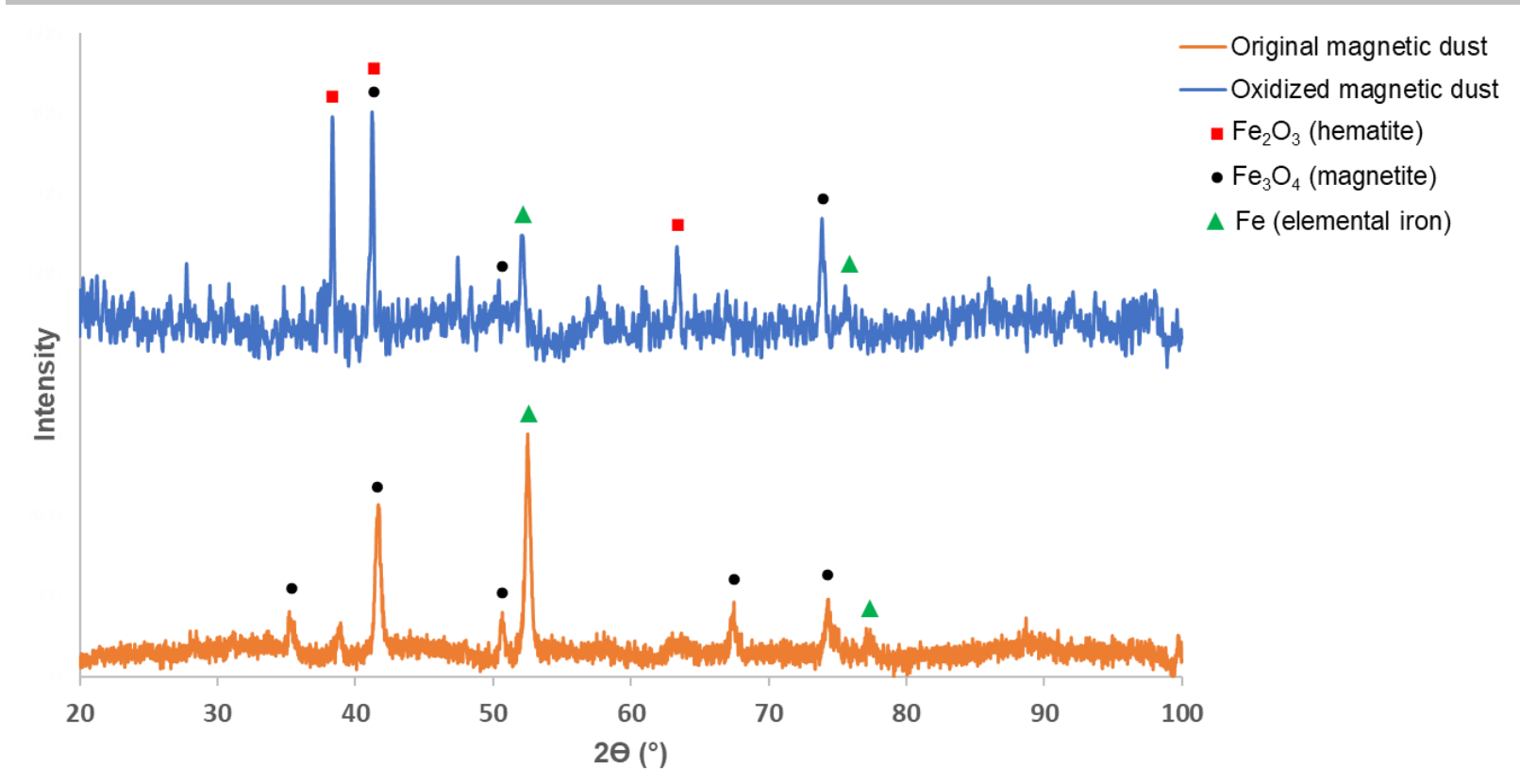

Figure S2: XRD patterns of original (orange) and oxidized (blue) magnetic dust. Decrease of $\mathrm{Fe}$ signal and increase of $\mathrm{Fe}_{2} \mathrm{O}_{3}$ signal suggest the oxidation of elemental iron.
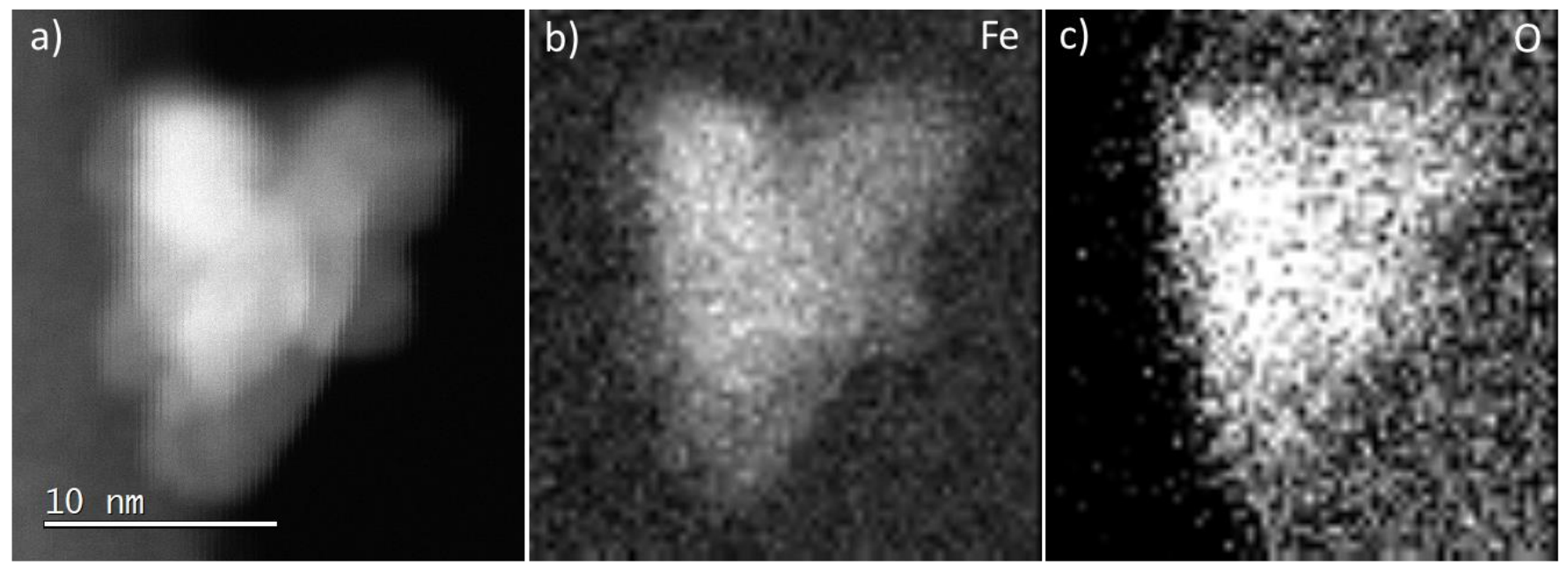

Figure S3: STEM-EELS elemental map for commercial magnetite nanoparticles. a) is an overview of the magnetite nanoparticle selected for elemental mapping; b) and c) are elemental maps for $\mathrm{Fe}$ and $\mathrm{O}$, respectively. 


\section{Supplementary Information}

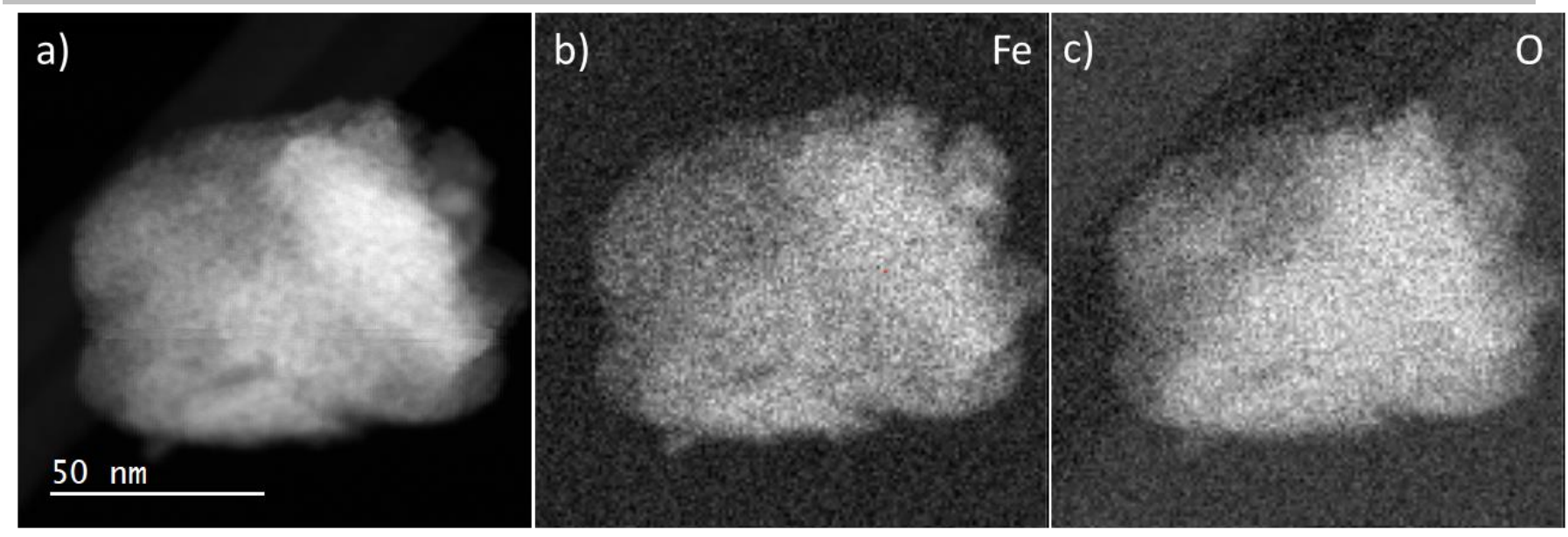

Figure S4: STEM-EELS elemental map for magnetite in dust particles without $\mathrm{Fe}^{0}$. a) is an overview of the dust nanoparticle selected for elemental mapping; b) and c) are elemental maps for $\mathrm{Fe}$ and $\mathrm{O}$, respectively.

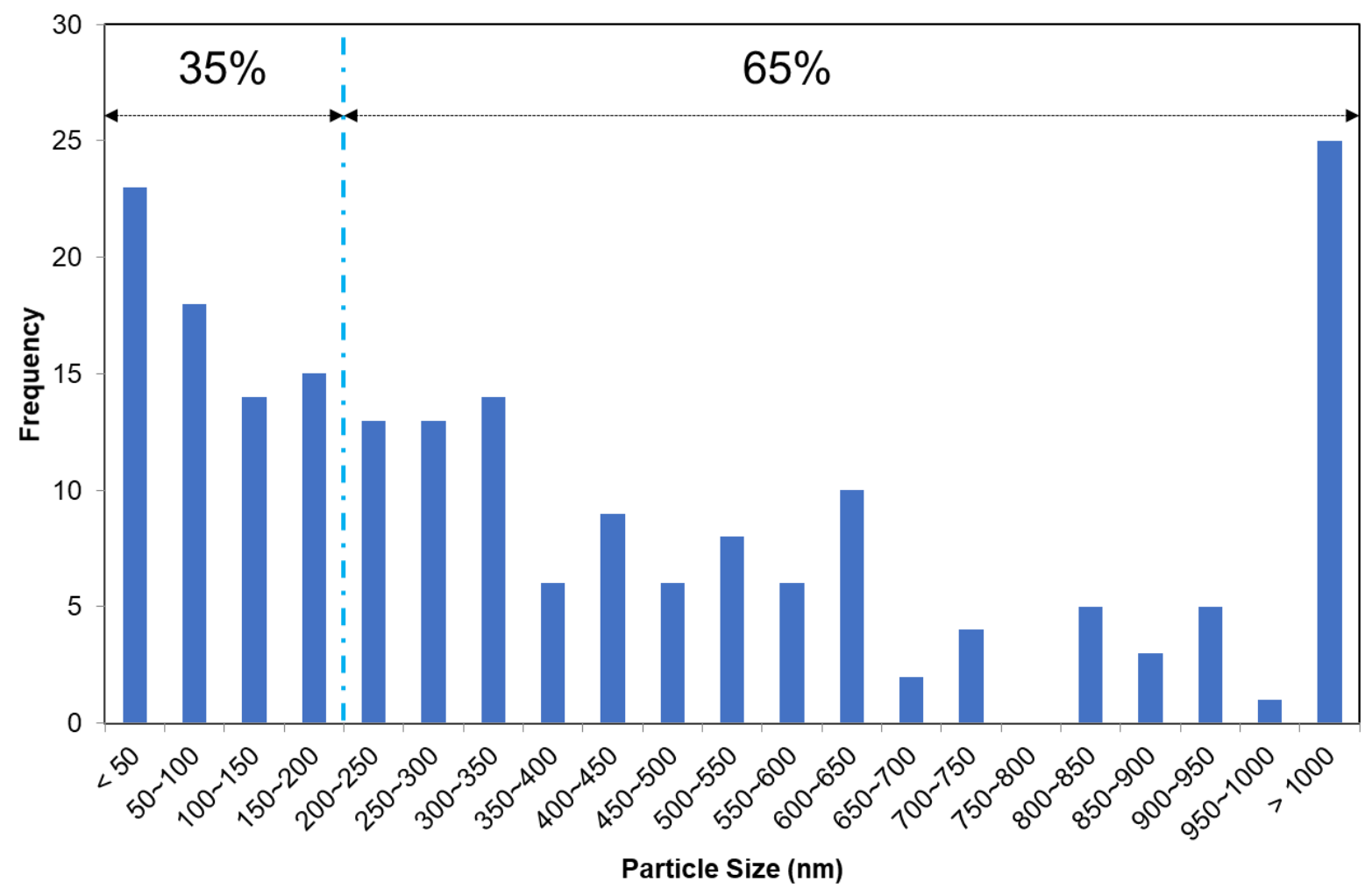

Figure S5: Magnetic dust particle size counting. 35\% of particles were smaller than 200 nm. 200 particles were counted using transmission electron microscope (TEM) to investigate particle size distribution. 


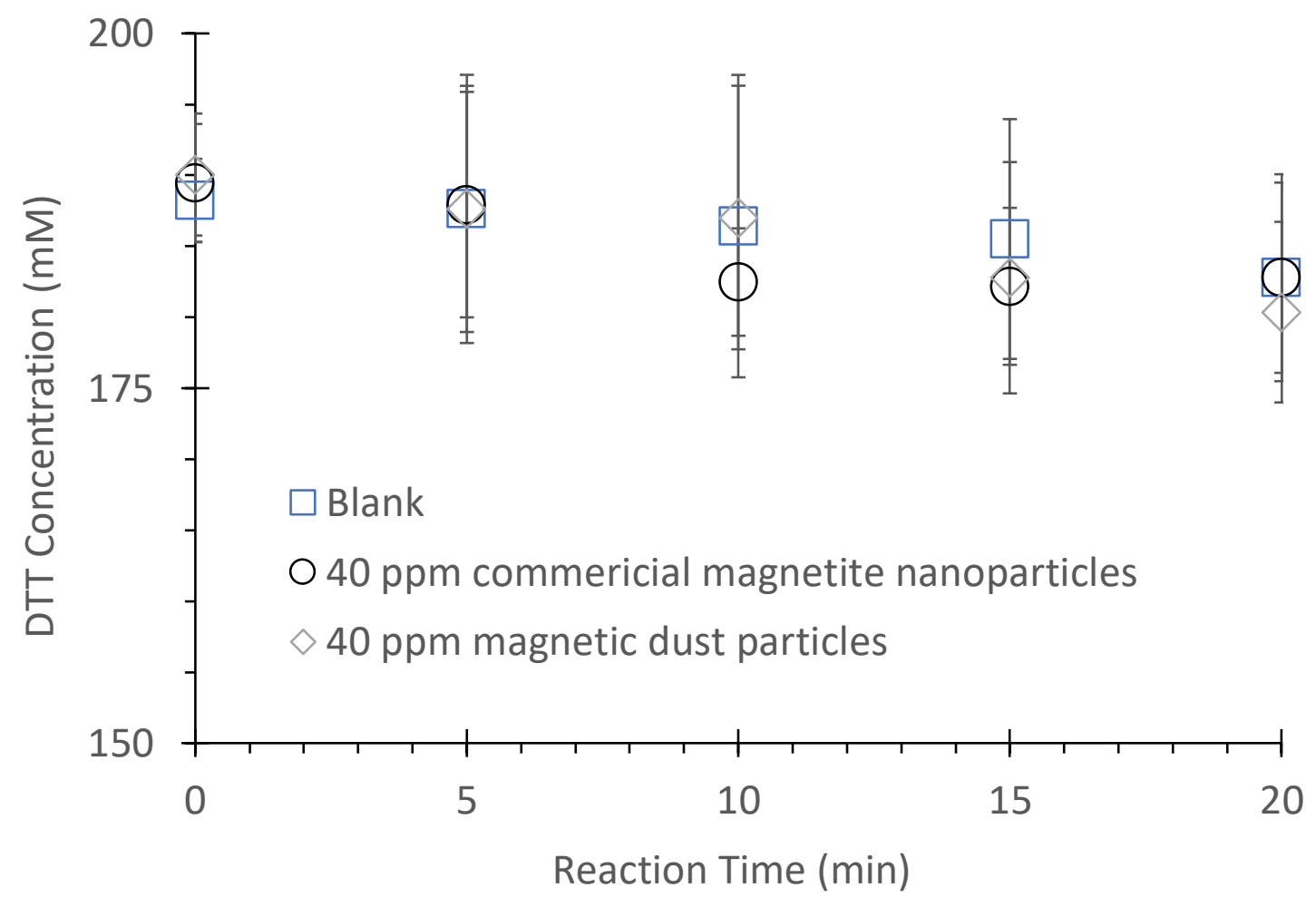

Figure S6: DTT assay for magnetite and magnetic dust. Negligible DTT activity indicates organic content in all samples is unlikely to induce ROS. A student t-test $(\alpha=0.1)$ concluded there is no statistically significant difference $(p=0.82)$ in the DTT depletion rates between magnetic dust particles and magnetite nanoparticles. 


\section{Supplementary Information}

\section{References}

(1) Ayton, S.; Wang, Y.; Diouf, I.; Schneider, J. A.; Brockman, J.; Morris, M. C.; Bush, A. I. Brain Iron Is Associated with Accelerated Cognitive Decline in People with Alzheimer Pathology. Mol. Psychiatry 2019. https://doi.org/10.1038/s41380-019-0375-7.

(2) Harischandra, D. S.; Rokad, D.; Neal, M. L.; Ghaisas, S.; Manne, S.; Sarkar, S.; Panicker, N.; Zenitsky, G.; Jin, H.; Lewis, M.; Huang, X.; Anantharam, V.; Kanthasamy, A.; Kanthasamy, A. G. Manganese Promotes the Aggregation and Prion-like Cell-to-Cell Exosomal Transmission of a-Synuclein. Sci. Signal. 2019, 12 (572), eaau4543. https://doi.org/10.1126/scisignal.aau4543.

(3) Zhang, C.; Liu, Z.; Zhang, Y.; Ma, L.; Song, E.; Song, Y. “ Iron Free " Zinc Oxide Nanoparticles with Ion-Leaking Properties Disrupt Intracellular ROS and Iron Homeostasis to Induce Ferroptosis. Cell Death Dis. 2020, 11 (3), 183. https://doi.org/10.1038/s41419-020-2384-5.

(4) Yen, C.; Harischandra, D. S.; Kanthasamy, A.; Sivasankar, S. Copper-Induced Structural Conversion Templates Prion Protein Oligomerization and Neurotoxicity. Sci. Adv. 2016, 2 (7), e1600014. https://doi.org/10.1126/sciadv.1600014.

(5) Zhong, M.; Zhang, Y. Melatonin Prevents Abnormal Mitochondrial Dynamics Resulting from the Neurotoxicity of Cadmium by Blocking Calcium-Dependent Translocation of Drp1 to the Mitochondria. J. Pineal Res. 2016, 60 (3), 291-302. https://doi.org/10.1111/jpi.12310.

(6) Mason, L. H.; Harp, J. P.; Han, D. Y. Pb Neurotoxicity: Neuropsychological Effects of Lead Toxicity. Biomed Res. Int. 2014, 2014, 840547.

https://doi.org/10.1155/2014/840547.

(7) Galván-Arzate, S.; Santamaría, A. Thallium Toxicity. Toxicol. Lett. 1998, 99 (1), 1-13. https://doi.org/10.1016/S0378-4274(98)00126-X.

(8) Pelclová, D.; Urban, P.; Ridzoň, P.; Ǎenholdová, Z.; Lukáš, E.; Diblík, P.; Lacina, L. Two-Year Follow-up of Two Patients after Severe Thallium Intoxication. Hum. Exp. Toxicol. 2009, 28 (5), 263-272. https://doi.org/10.1177/0960327109106487. 